\title{
Analysis of the Density and Distribution of HER2 In Breast Cancer Cell Subpopulations and Their Response to Anti-Cancer Drugs on the Single Cell Level Using Liquid-Phase Electron Microscopy
}

\author{
Patricia Blach ${ }^{1,2^{*}}$, Florian Weinberg ${ }^{1}$, Diana Peckys ${ }^{3}$ and Niels de Jonge ${ }^{1,2}$ \\ 1. INM - Leibniz Institute for New Materials, D-66123 Saarbrücken, Germany \\ 2. Department of Physics, Saarland University, D-66123 Saarbrücken, Germany \\ 3. Department of Biophysics, Saarland University, D-66421 Homburg, Germany \\ * Corresponding author: Patricia.Blach@leibniz-inm.de
}

Breast cancer is known to be the leading cause of mortality in women worldwide. According to the presence of growth factor receptors on the surface of the cells it is divided into different subtypes [1]. HER2 is a growth factor receptor which belongs to the EGF-receptor family and is overexpressed in around $20 \%$ of all breast cancer cases [2]. HER2 positive breast cancer can nowadays be treated with neutralizing antibodies (Trastuzumab or Pertuzumab) or small compound inhibitors (Lapatinib), but the development of drug resistances remains a big problem in clinics [3]. Our aim is to understand the mechanism of trastuzumab treatment as well as the development of drug resistances. As individual cancer cells correspond differently towards therapy, we are interested in the action of trastuzumab on the single cell level [4].

HER2 was analyzed on the surface of cancer cells by labeling 1:1 with a biotinylated affibody followed by Trastuzumab treatment for different time points and incubation with streptavidin-conjugated quantum dot nanoparticles. Additionally, cells were co-stained with CD44 as a marker for stem cell subpopulation. Fluorescence microscopy was performed on the labeled cells followed by scanning transmission electron microscopy (STEM) analysis with nanometer resolution. Images were acquired from flat and ruffled surface regions of the cells. Exemplary images are shown in Figure 1. The location of the quantum dots provides information about the spatial distribution of the HER2 receptors. Automatic label detection was used to analyze the distribution by pair correlation function analysis. HER2 was shown to be either present as single molecules or homo-dimers which is the cell growth signaling active state.

Figure 1c shows the pair correlation function of QD-labeled HER2 in ruffled, flat and stem-like cells. The peak at 20-nm distance, indicating the presence of HER2 homo-dimers, was more pronounced in ruffled regions while in flat- and stem-like cancer cells, the occurrence of this inter-label distance stayed in the range of a random distribution. Incubation with the tested drug influenced the dimer peak in the pair correlation function. After 3 and 10 minutes of trastuzumab treatment, the presence of HER 2 on the cell surface was already significantly reduced as well as the number of homo-dimers (Figure 1d). The dimerization of the receptors was shown to vary in different parts of the cell. This suggests an influence of cell heterogeneity on the function of the receptors and hence on the drug response. The wet samples were coated with graphene to keep the cells in hydrated state during STEM imaging. Beam induced radiation damage dose series were performed on these samples (Figure 2a, b). Therefore, the distance between two particles was measured for the respective electron doses applied to the sample. As seen in Figure $2 c$, no change was found in any of the samples up to a dose of $(2.1 \pm 0.08) \times 10^{3} \mathrm{e}^{-} / \AA^{2}$.

With our established protocols we are able to investigate HER2 distribution on the single cell level. It was 
also shown that graphene coated cells were resistant to radiation damage during an electron beam irradiation [5].

\section{References:}

[1] G Turashvili and E Brogi, Frontiers in medicine 4 (2017), p. 227.

[2] PR Pohlmann, IA Mayer and R Mernaugh, Clinical cancer research : an official journal of the American Association for Cancer Research 15 (2009), p. 7479.

[3] T Vu and FX Claret, Frontiers in oncology 2 (2012), p. 62.

[4] DB Peckys et al., Molecular biology of the cell 28 (2017), p. 3193.

[5] We thank Eduard Arzt for his support through INM. This work was funded by the Else KrönerFresenius Stiftung.
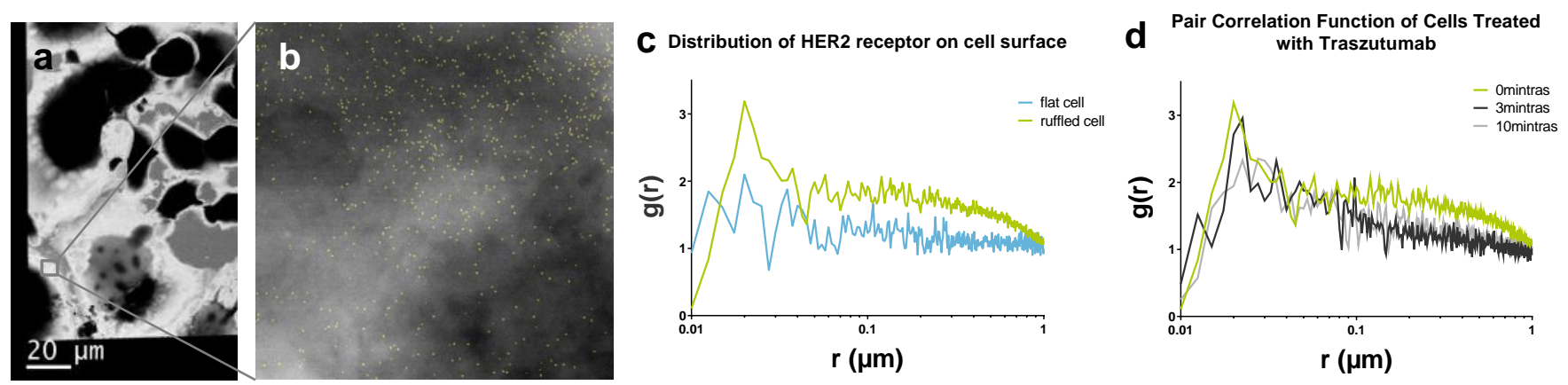

Figure 1. STEM images of HER2 labeled breast cancer cells at $200 \mathrm{kV}$. a) Exemplary STEM overview image at 800x magnification. b) Image of quantum dot labels at 80 000x magnification and an electron dose of $39 \pm 1 \mathrm{e}^{-} / \AA^{2}$. Yellow spots indicate the automatic detection of the quantum dots. c) Pair correlation function $\mathrm{g}(\mathrm{r})$ of a ruffled $(\mathrm{N}=1324$ labels $)$ and flat $(\mathrm{N}=956$ labels $)$ cell indicating HER2 distribution and homo-dimer formation at a center-to-center label pair distance $r=20 \mathrm{~nm}$ for the ruffled cell. d) Pair correlation function $\mathrm{g}(\mathrm{r})$ of trastuzumab treated cells showing reduced probability of the $20 \mathrm{~nm}$ peak for incubation times of $10 \mathrm{~min}$. For $0 \min \mathrm{N}=1324$ labels, $3 \min \mathrm{N}=883$ labels and for $10 \min \mathrm{N}=856$ labels.
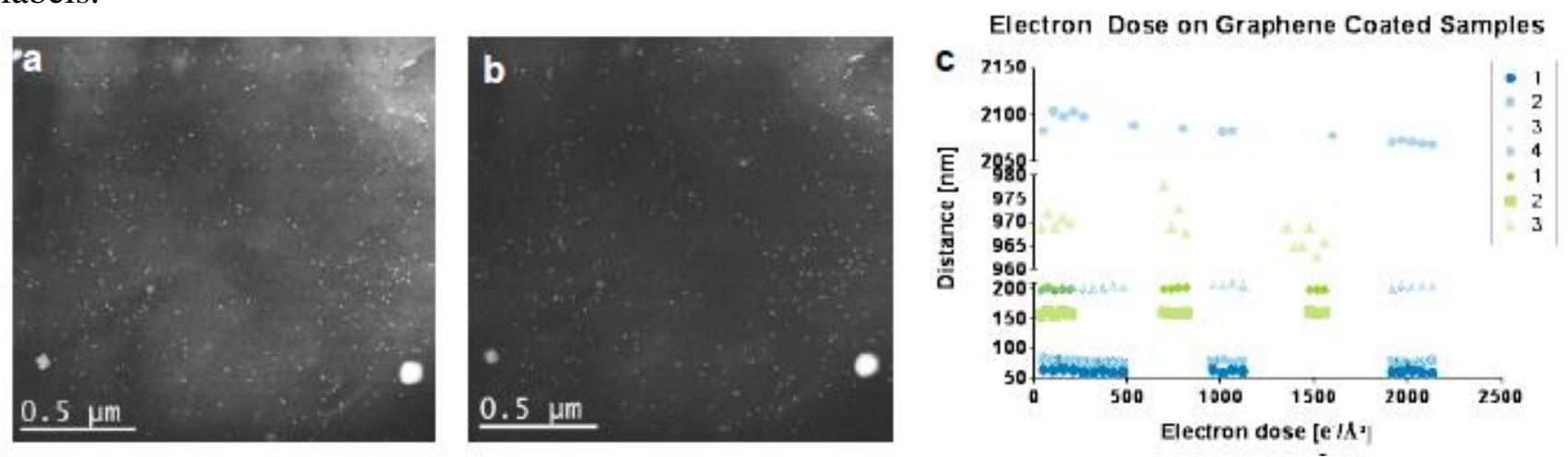

Figure 2. Radiation damage measurement. a) First image of the dose series $\left(53 \pm 2 \mathrm{e}^{-} / \AA^{2}\right.$ for the blue dots and $39 \pm 1 \mathrm{e}^{-} / \AA^{2}$ for the green dots). b) Last image of the dose series $(2.1 \pm 0.08) \times 10^{3} \mathrm{e}^{-} / \AA^{2}$ for $1 \mathrm{C}$ and $(1.5 \pm 0.04) \times 10^{3} \mathrm{e}^{-} / \AA^{2}$ for $\left.2 \mathrm{C}\right)$. c) Electron dose of graphene coated samples showing no change in distances between two particles up to an electron dose of $(2.1 \pm 0.08) \times 10^{3} \mathrm{e}^{-/ \AA^{2}}$. Images were acquired at $200 \mathrm{kV}$ at the JEM-ARM200F. 